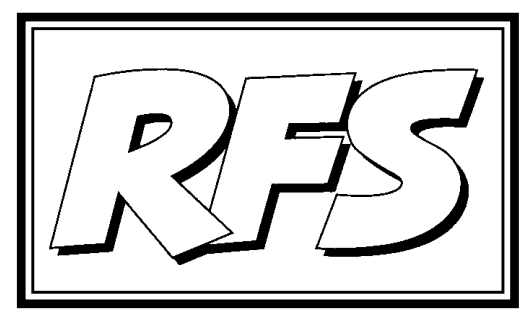

Revista de Fomento Social, 59 (2004), 685-698

\title{
Universidad y Cooperación al Desarrollo
}

\author{
UNIJES
}

Nota previa.- El presente texto constituye la reflexión de una Comisión de UNIJES (Federación de Centros Universitarios de la Compañía de Jesús en España ${ }^{1}$ ) que, en su original, lleva por título "Bases para la cooperación al desarrollo en los centros universitarios de la Compañía de Jesús en España". Lo hemos considerado de interés general para nuestros lectores, en particular para aquellos preocupados por esta dimensión de la actividad universitaria.

La cooperación al desarrollo de los centros universitarios de la Compañía de Jesús debe entenderse articulando diferentes coordenadas. Ante todo, desde la identidad de la institución que actúa: un centro de la Compañía de Jesús, que tiene además carácter universitario. En segundo lugar, con una adecuada comprensión de lo que es el desarrollo de los pueblos, de cómo orientar la cooperación, y de cómo hacer todo eso desde una institución

1 Véase más información en la página Web de UNIJES: < $\underline{\text { www.unijes.net }>}$ 
universitaria. Todo ello nos permitirá llegar a algunas conclusiones sobre la organización y funcionamiento de la cooperación al desarrollo en nuestros centros y sobre la colaboración entre ellos en este terreno.

\section{El marco: una institución universitaria de la Compañía de Jesús}

Lo que es una institución universitaria de la Compañía de Jesús debe marcar todas las tareas que se realizan en ella, y concretamente la cooperación al desarrollo. Y los rasgos esenciales a dichas instituciones fueron expresados por la Congregación General 34 (1995), a partir de la experiencia de cuatro siglos, de la siguiente manera:

... mantenery aun fortalecer el carácter específico de cada una de nuestras instituciones: en cuanto jesuítica y en cuanto universidad. Pensando en el futuro, tenemos que estar muy alerta para que tanto el sustantivo "universidad" como el adjetivo "jesuítica" sean siempre plenamente respetados ${ }^{2}$.

Es esencial que una institución universitaria de la Compañía de Jesús responda a las exigencias propias de toda universidad. En palabras de la "Carta Magna de las Universidades Europeas" suscrita en Bolonia en 1988, una universidad ha de ser

... una comunidad académica que, de modo riguroso y crítico, contribuye a la tutela $\boldsymbol{y}$ desarrollo de la dignidad humana y de la herencia cultural mediante la investigación, la enseñanza y los diversos servicios ofrecidos a las comunidades locales, nacionales e internacionales.

Es, por tanto, la actividad en estas tres líneas de trabajo la que define el auténtico quehacer universitario: investigación, enseñanza y servicios. Y así se lo propone también la Compañía de Jesús en la Congregación General citada:

El sustantivo [universidad] garantiza el compromiso con la autonomía fundamental, la integridad y la sinceridad de una universidad, precisamente en cuanto universidad: un lugar de serena y abierta investigación y discusión de la verdad. Al mismo tiempo, indica los objetivos propios de toda universidad (investigación, enseñanza, diversos servicios consecuentes a su misión cultural) como horizonte y contexto indispensable para la auténtica conservación, renovación y comunicación del saber y de los valores humanos $^{3}$.

Pero además una institución universitaria de la Compañía de Jesús o que funciona bajo su última responsabilidad es jesuítica: y eso

2 Congregación General 34, Decreto 17: La Compañía y la vida universitaria, n. 5.

3 Ibid., n. 6. 
... significa la participación genuina (...) en la identidad y misión fundamentales de la Compañía. Aunque queremos evitar toda instrumentalización de la universidad o la reducción de su misión a una única meta legítima, el adjetivo 'jesuítica', con todo, requiere de la universidad armonía con las exigencias de servicio de la fe y promoción de la justicia establecidas por la Congregación General 32, decreto 4. La universidad jesuítica puede y debe descubrir en su propia contextura institucional y en sus genuinos objetivos un ruedo específico y adecuado para el encuentro con la fe que obra la justicia ${ }^{4}$.

Más concretamente, el P. General, Peter-Hans Kolvenbach, remontándose a los primeros tiempos de la Compañía de Jesús, sintetizaba la misión de un centro universitario jesuítico en cuatro puntos ${ }^{5}$ :

- la excelencia académica, con buenos profesionales que aportan conocimientos y los "bienes internos" vinculados a su profesión;

- el servicio a la construcción de una sociedad más justa y más humana, abierta a la trascendencia y a la fe ${ }^{6}$;

- la creación de conocimiento y el gusto por transmitirlo a los alumnos;

- el facilitar a sus miembros la apertura a la trascendencia, la búsqueda de sentido.

La tarea del desarrollo de los pueblos conecta profundamente con la misión de la Compañía de Jesús hoy. Es más, la conciencia de las diferencias Norte-Sur entendida como injusticia estructural está en el origen mismo de la redefinición que la Compañía ha hecho de su misión en los años recientes. De ahí la importancia de definir cuál ha de ser la responsabilidad específica de los centros universitarios jesuitas en relación con la cooperación al desarrollo.

5 Lo hacía en la Alocución a la Reunión Internacional de la Educación Superior de la Compañía, sobre "La Universidad de la Compañía de Jesús a la luz del carisma ignaciano", Roma 27 de mayo de 2001.

6 Lo expresaba de forma brillante y muy sugestiva Ignacio Ellacuría en su discurso en la Universidad de Santa Clara (1982): "Una universidad cristiana tiene que tener en cuenta la preferencia del evangelio por el pobre. Esto no significa que sean los más pobres los que deban entrar a cursar sus estudios en la Universidad, ni que la Universidad deba dejar de cultivar toda aquella excelencia académica que se necesita para resolver los problemas reales que afectan a su contexto social. Significa más bien que la Universidad debe encarnarse entre los pobres intelectualmente para ser ciencia de los que no tienen voz, el respaldo intelectual de los que en su realidad misma tiene la verdad y la razón, aunque sea a veces a modo de despojo, pero no cuentan con las razones académicas que justifiquen y legitimen su verdad y su razón". 


\section{Cooperación al desarrollo}

Para definir lo que debe ser nuestra cooperación universitaria al desarrollo tenemos que clarificar previamente dos conceptos: el de desarrollo y el de cooperación al desarrollo.

\subsection{Desarrollo}

No cabe identificar desarrollo con crecimiento económico; ni, por consiguiente, medir el nivel de desarrollo por la renta per cápita; ni poner la clave de la estrategia para el desarrollo en la inversión y la capacidad de ahorro.

Frente a esas concepciones, desde finales de los años ochenta se perfilan planteamientos alternativos que sitúan la lucha contra la pobreza como centro prioritario de toda acción contra el subdesarrollo y señalan a la sociedad civil como el agente principal del desarrollo.

En dicho sentido, la lucha contra el subdesarrollo no busca paliar los efectos de este, sino directamente atajar las causas que lo producen.

El auténtico desarrollo ha de ser definido entonces

- como desarrollo humano: en el sentido del Programa de Naciones Unidas para el Desarrollo, como el "proceso de ampliar la gama de opciones de las personas, brindándoles mayores oportunidades de educación, atención médica, ingreso y empleo, y abarcando el espectro total de opciones humanas, desde un entorno físico en buenas condiciones hasta libertades económicas y políticas" (PNUD 1992);

- como desarrollo sostenible: en el sentido de la Comisión Mundial del Medio Ambiente y Desarrollo ONU, como "aquel que satisface las necesidades del presente sin limitar el potencial para satisfacer las necesidades de las generaciones futuras".

En palabras de Amartya Sen, el desarrollo ha de ser entendido como "el proceso de expansión de las libertades reales de que disfrutan los individuos".

No es posible olvidar los obstáculos procedentes del actual orden económico mundial, que son responsabilidad en primer lugar de los países más desarrollados. Pero, por lo que atañe a la responsabilidad de los mismos países en desarrollo, este proceso no puede ser entendido sólo a nivel individual, ni sólo promovido desde el Estado o desde agentes externos. 
Un verdadero desarrollo pasa hoy por la movilización de la sociedad civil y tiende, en fin de cuentas, al progreso social. Esto significa que el desarrollo ha de ser integral y endógeno:

- integral, porque el progreso ha de ser buscado simultánea y articuladamente en los terrenos económico, social, político, cultural y moral, de forma que todos ellos se refuercen entre sí;

- endógeno, porque este proceso multisectorial debe buscarse desde la propia sociedad, desde su capacidad para actuar sobre sí misma, tomando conciencia de sus potencialidades y promoviendo unas mejores condiciones de vida y una participación democrática.

\subsection{Cooperación al desarrollo}

Desde finales de la segunda guerra mundial, muchos Estados del primer mundo pusieron en práctica una política de ayuda al desarrollo que se caracterizaba por los siguientes rasgos: a) estaba protagonizada esencialmente por los gobiernos; b) era un instrumento al servicio de la política exterior de estos; c) se basaba en el convencimiento de que el crecimiento económico conduciría automáticamente a un mayor bienestar de la población.

Estos enfoques han sido puestos en cuestión posteriormente, en tres direcciones: a) se ha revisado el concepto de desarrollo en los términos ya mencionados; b) la sociedad civil ha asumido un creciente protagonismo en todas estas tareas; c) se ha sustituido la ayuda por una verdadera cooperación.

Por cooperar entiende el Diccionario de la Real Academia de la Lengua: "obrar conjuntamente con otro u otros para un mismo fin". La cooperación al desarrollo concibe las relaciones entre las partes implicadas de forma radicalmente distinta a como lo hacía la ayuda al desarrollo.

La ayuda es unidireccional; la cooperación supone que las dos partes trabajan juntas en torno a intereses comunes: los agentes del Norte aportan recursos humanos, económicos y tecnológicos, mientras que los agentes del Sur colaboran en la definición de las necesidades y en la ejecución de las acciones. Porque, si el desarrollo se basa en la emergencia de la sociedad civil, la cooperación al desarrollo implica confiar en las capacidades de los "beneficiarios" para decidir lo que les conviene. 


\section{Cooperación universitaria al desarrollo}

Si la universidad ha sido históricamente un agente de progreso, tanto por su labor formativa como por su papel dinamizador y movilizador de la sociedad, el desarrollo y la cooperación al desarrollo, tal como acaban de ser presentados, ofrecen oportunidades inmensas de acción para una institución universitaria.

\subsection{Algunos rasgos específicos de la cooperación universitaria al desarrollo}

Las instituciones universitarias, en general, se han incorporado sólo recientemente de forma oficial a la tarea de la cooperación al desarrollo. Esta incorporación ha suscitado recelos e incluso oposición de otros actores, especialmente no oficiales, que ya jugaban un papel en este campo.

Sin embargo, la complementariedad y pertinencia de los centros universitarios en la cooperación al desarrollo respecto de otros actores está fuera de toda duda. Por sus propias características están llamadas a jugar un papel de catalizador y apoyo para la acción de otras instituciones de desarrollo.

Entre estas características propias de la cooperación universitaria al desarrollo conviene citar:

$1^{\circ}$ Como en toda iniciativa de cooperación al desarrollo, el centro ha de ser la persona humana; más concretamente, las personas que sufren situaciones de exclusión (racial, económica, social, de género...), los pobres o empobrecidos por el sistema socioeconómico, víctimas de la violencia, de la marginación o de la injusta distribución de los bienes y de las oportunidades...

$2^{\underline{ }}$ Sin embargo, las instituciones universitarias son, por su propia naturaleza, instituciones de vía lenta ${ }^{7}$ : ello explica que su acción no tenga, muchas veces, efectos directos e inmediatos sobre las víctimas del subdesarrollo.

$3^{\text {o }}$ Ahora bien, ese carácter hace a las instituciones universitarias más idóneas para una acción estructural, que arranque de la identificación del complejo sistema de causas globales, nacionales, regionales, locales y también personales. Y es evidente que la identificación de los proble-

7 I. GonZÁlez Modroño, Intervención en las Jornadas Interuniversitarias del Profesorado de COCESU (Loyola 2002). 
mas, su caracterización, evaluación y el análisis de sus causas parece ser previo a la realización de cualquier actividad de desarrollo.

$4^{\circ}$ Este tipo de actuación permite a las instituciones universitarias generar y difundir un acervo de conocimientos e interpretaciones de la realidad desde la perspectiva de los empobrecidos, que contrasta con la lógica del "pensamiento único". En esta generación de pensamiento alternativo, las instituciones universitarias están perdiendo importancia frente a otros actores que están trabajando intensamente en el análisis de los problemas medioambientales, del desarrollo humano o del sistema socioeconómico.

$5^{\circ} \mathrm{Al}$ comprometerse en esta línea, los centros universitarios de la Compañía de Jesús tienen que aceptar el reto de saberse no completamente dentro del marco de la excelencia académica y científica, tal como es entendida hoy en el mundo universitario en general. Esa "excelencia" se mide por las páginas publicadas en las revistas que figuran en los índices internacionales, donde ciertos temas suelen quedar excluidos porque no tienen interés para las tendencias científicas internacionales dominantes. No se trata de renunciar a la excelencia, sino de promover un concepto de la misma que incluya entre sus valores la capacidad de afrontar el cambio social.

Si asumimos consecuentemente esta forma de ser en la cooperación, se abrirá para nosotros una triple perspectiva:

a) Un mayor enriquecimiento para nuestras propias instituciones universitarias, que quedarán marcadas por esas realidades diferentes a las que conforman el ambiente normal de nuestros centros.

b) La posibilidad de colaboración con otras instituciones no universitarias en este campo del desarrollo desde nuestra propia especificidad. Entre ellas habrá que contar en primer lugar con otras obras de la Compañía de Jesús o vinculadas a esta, tanto en el campo de la cooperación (Intermón, Entreculturas - Fe y Alegría, Alboan, Servicio Jesuita de Refugiados...) como en el de la reflexión e incidencia (Grupo Fomento Social, Internacional Jesuit Network for Development...).

c) La posibilidad de trabajo en red con otras instituciones universitarias de la Compañía de Jesús (especialmente con AUSJAL). 


\subsection{Los objetivos de la cooperación universitaria al desarrollo}

De acuerdo con todo lo que precede, tres objetivos generales pueden guiar la cooperación universitaria al desarrollo:

1. La contribución a la formación de capital humano del Sur y en el Sur.

2. El apoyo al fortalecimiento de las instituciones de los países del Sur, especialmente de las universitarias (porque no se construye país sin construir universidad).

3. La presencia de la cooperación al desarrollo en el día a día de la docencia y la investigación en nuestros centros universitarios del Norte.

Los dos primeros objetivos generales de la cooperación al desarrollo tienen su campo directo de actuación en los países del Sur: buscan el fomento del desarrollo del mundo empobrecido a partir del aprovechamiento del efecto multiplicador de la formación de formadores y de la mejora de las capacidades y recursos de las universidades contrapartes. El tercer objetivo general tiene, en cambio, su campo de acción en nuestro país.

\subsection{La cooperación universitaria al desarrollo: tareas en el Sur}

Corresponde a los dos primeros objetivos generales enunciados antes. Podemos concretarlos ahora a unos objetivos más específicos:

- Formación del capital humano con mentalidad y valores de compromiso social: transmisión de conocimientos, valores y destrezas.

- Formación de formadores buscando un efecto multiplicador en la sociedad de cada país.

- Transmisión de experiencia docente y de tecnología educativa.

- Desarrollo de capacidades institucionales y transmisión de herramientas de gestión universitaria.

- Transmisión de tecnología específica en el campo del desarrollo económico y social.

— Colaboración en el diseño y ejecución de proyectos integrales de desarrollo.

— Transferencia de financiación. 
Si abordamos ahora la secuencia temporal de la cooperación al desarrollo y la aplicamos a una institución universitaria, cabe distinguir tres fases:

Primera fase: diagnóstico. Como en cualquier proyecto de cooperación al desarrollo, los primeros pasos deben ir en la línea de construir un diagnóstico suficientemente completo para definir los objetivos, contenidos y prioridades de la cooperación universitaria. Para ello es preciso la intervención de una contraparte adecuada, que actúe desde aquella realidad, y que en nuestro caso puede ser preferentemente una universidad (jesuita o no).

Segunda fase: formación de formadores para garantizar un profundo efecto multiplicador. Este efecto multiplicador puede buscarse por dos vías complementarias: una personal y otra institucional. La vía personal comprende la formación de profesores en el país de origen y los periodos de formación de postgrado de los profesores en España. La vía institucional se orienta a la mejora de las capacidades docentes e investigadoras de centros similares en los países en desarrollo, para que ellos mismos puedan responder más adecuadamente a la demanda de la sociedad de expertos, técnicos o gestores, y afrontar más rigurosamente los problemas que frenan el desarrollo del país y la lucha contra la pobreza. Por último, y en la medida de lo posible, estos esfuerzos deben ir complementados por el apoyo a la gestión universitaria mediante transferencia de modelos de gestión y apoyo financiero para la mejora de las condiciones docentes. Si esta tarea de formación de formadores es efectiva, se cumplirá con uno de los objetivos de la cooperación al desarrollo: dejar de ser necesarios, en la medida en que, paulatinamente, los profesionales formados terminan cubriendo los huecos en la formación que inicialmente son asumidos por el centro universitario del Norte.

Tercera fase: formulación de políticas y creación de estructuras. Estas actuaciones consisten básicamente en la colaboración con gobiernos, administraciones y agentes sociales para la creación de estructuras y formulación de políticas de desarrollo de sus países, a partir de los conocimientos y el capital humano generados en la fase anterior. Si el trabajo realizado en esa fase anterior ofrece como doble resultado la presencia de capital humano formado en las universidades y la experiencia del trabajo conjunto, están creadas las bases para equipos conjuntos de capital humano que trabajen en proyectos de formulación de políticas estatales, creación de estructuras de formación, asesoramiento a los agentes públicos y privados del país, ejecución de programas y proyectos. 


\subsection{La cooperación universitaria al desarrollo: tareas en el Norte}

El tercer objetivo general (la presencia de la cooperación al desarrollo en el día a día de la docencia y la investigación en nuestros centros universitarios del Norte) expresa el efecto retorno que la cooperación universitaria al desarrollo tiene para el centro universitario que lo pone en práctica. En este sentido, la cooperación al desarrollo no es sólo unidireccional: tiene también efectos muy positivos para los agentes del Norte. Dicho efecto retorno se traduce en un cúmulo de conocimientos y de experiencias de primera mano de una realidad diferente, así como en una sensibilización nueva a toda esa problemática, que afecta en primer lugar a los que intervienen directamente en esas tareas. Pero esta sensibilidad terminará afectando también a la institución universitaria toda y podrá concretarse en estos objetivos específicos:

- Impregnar las actividades universitarias en general, docentes o de investigación, de esta preocupación por las desigualdades mundiales y las injusticias que de ahí derivan.

- Investigar en temas relacionados con el desarrollo.

- Organizar cursos, en las enseñanzas regladas (asignaturas de libre configuración, seminarios) o en las propias (masters y otros cursos de postgrado), sobre cuestiones relacionadas con el desarrollo de los pueblos.

- Sensibilizar en nuestros centros del primer mundo y su entorno con actividades de difusión, experiencias de voluntariado, etc.

- Colaborar con organizaciones no gubernamentales de desarrollo (ONGD) de España y asesorarlas en la elaboración, ejecución y evaluación de sus proyectos.

\section{Lugar de la cooperación al desarrollo en nuestras instituciones universitarias}

En tiempos de crisis de las instituciones universitarias españolas, afectadas tanto por la caída demográfica como por el aumento de la competencia nacional o los cambios en ciernes a nivel europeo, parece especialmente difícil plantear cambios en sus estructuras (especialmente si eso supone la creación de un nuevo "lugar").

Sin embargo, por coherencia con nuestra misión y también por necesidad 
de reforzar nuestra propia identidad, parece urgente dar los primeros pasos para establecer la actividad de cooperación universitaria al desarrollo en todos nuestros centros de manera eficaz, sostenible e integrada con el resto de la actividad universitaria.

Para ello hay que contar con la experiencia de cada centro en este campo y respetar sus peculiaridades. Con el deseo de diseñar un modelo compartido, aunque no idéntico para todos (porque nuestros centros no son homogéneos, ni por dimensiones ni por otros muchos rasgos históricos y actuales), señalamos algunas características del mismo así como la infraestructura mínima y los recursos humanos y económicos necesarios.

\subsection{Características organizativas}

La cooperación universitaria al desarrollo debe funcionar según estas características:

$1^{\text {a }}$ Integrada en las funciones universitarias, concretamente con capacidad para la docencia, la investigación y la extensión y servicios (proyectos).

$2^{\mathrm{a}}$ Multidisciplinar, de forma que permita abordar tanto los proyectos como las investigaciones o la docencia desde múltiples enfoques e integrar dichas visiones.

$3^{\text {a }}$ Interfacultativa: abierto a la participación de profesores e investigadores de otros departamentos, institutos, facultades y escuelas de la institución.

$4^{\mathrm{a}}$ Con capacidad para dinamizar a la comunidad universitaria: abierta a la participación de alumnos, personal, ex-alumnos y otros miembros de la comunidad universitaria.

$5^{\text {a }}$ Diferenciada del área de relaciones internacionales del centro (ya que estas no siempre tienen por objeto la cooperación al desarrollo), pero articulada con ellas para aprovechar sinergias y otras oportunidades.

6 ${ }^{\text {a }}$ Participativa: de forma que participen directamente en su gestión y en sus actividades, no sólo la propia comunidad universitaria, sino también asociaciones, fundaciones e incluso empresas.

$7^{\text {a }}$ Dotada de medios humanos y materiales suficientes, en función de la misión y la visión de la institución y de sus objetivos a medio y largo plazo. 


\subsection{Infraestructura mínima}

Para garantizar el cumplimiento de la misión de cooperación universitaria al desarrollo sería necesario dotar a esta área de una entidad propia y diferenciada dentro de la institución universitaria, con capacidades tanto de docencia como de investigación y de servicios.

Cooperación al desarrollo deberá contar con un responsable, que no tenga simultáneamente otras tareas funcionales, y con unos locales propios, que la doten de la necesaria visibilidad. En ese lugar desarrollará su actividad el personal que le sea asignado.

Cooperación al desarrollo estará dotada de la autonomía necesaria para organizarse y funcionar, de forma que se disponga de la necesaria flexibilidad para la realización de propuestas y proyectos y para la organización de sus actividades. Al mismo tiempo deberá tener un claro vínculo de dependencia respecto a la máxima autoridad de la institución, como expresión de la centralidad de la cooperación al desarrollo dentro de la misión del centro.

\subsection{Recursos humanos}

Aparte del responsable del área y de las personas asignadas a ella, según se ha dicho ya, se deberá facilitar en lo posible la participación del profesorado, investigadores y otro personal en proyectos y actividades de cooperación al desarrollo. Para ello se deberá proveer de los medios humanos, económicos y materiales necesarios que permitan esta participación, garantizando el principio de "participación no onerosa y no lucrativa". En concreto, será necesario garantizar:

- La posibilidad de incorporarse a proyectos de desarrollo (ya sean docentes, de investigación o de extensión) por parte de personal que así lo desee, siempre en coordinación con el centro en que presta su servicio habitual, reconociendo para ello oficialmente los tiempos de dedicación a actividades de cooperación.

- Un régimen de sustitución académica que evite la acumulación de clases pendientes para aquellos profesores que realicen viajes en el marco de proyectos de cooperación.

- Condiciones de viajes, alojamiento y manutención dignas, pero no lucrativas para el participante. 
- Valoración en la carrera profesional dentro de la institución universitaria de estas actividades, respecto de los proyectos de investigación, publicaciones, actividades académicas, etc., de forma que no se aparte de la carrera académica a aquellas personas comprometidas con la cooperación universitaria al desarrollo.

Será deseable también la participación de los alumnos, que podría realizarse según distintas modalidades:

- En actividades académicas (con reconocimiento de créditos, si es aplicable) de educación para el desarrollo, facilitando la realización de trabajos de investigación (tesinas, proyectos de carrera y tesis doctorales) en este área, ya sean desde una perspectiva temática o multidisciplinar.

- En actividades de voluntariado (en ocasiones también convalidables como prácticas), con experiencias de cooperación en el extranjero, de colaboración con ONGD en el ámbito universitario, etc., desde el convencimiento de que estas actividades tienen una alta capacidad educativa para los que las realizan.

No cabe olvidar tampoco en este apartado la necesidad de programas de becas de grado y postgrado para alumnos de países en desarrollo, que deberían aumentar en número y dotación, pudiéndose para esto además aprovechar bien la colaboración con la red de centros jesuitas en el mundo.

\subsection{Recursos económicos}

Cooperación al desarrollo no debe ser concebida como una fuente de costes: ha de tener capacidad para generar fondos, creando "productos" capaces de atraer recursos públicos y privados para financiar actividades de cooperación. Dichos recursos existen, aunque fluctúan según las políticas gubernamentales de cada momento: por eso hay que saber cómo atraerlos, buscando incluso la fórmula jurídica más idónea.

La aportación económica de la propia institución suele ser mucho mayor de lo que se puede cuantificar. Nunca hay que considerarla como un gasto: es una verdadera inversión porque genera, no sólo fondos complementarios, sino verdaderos activos intangibles para la institución. En todo caso, es deseable alguna aportación económica explícita como un signo inequívoco del compromiso con esta tarea: podría ser el $0,7 \%$ del presupuesto de la institución, u otra cantidad según las posibilidades de cada centro. 
La gestión financiera de la cooperación al desarrollo debe estar dotada de la autonomía necesaria para moverse en ese campo, que no siempre se adapta a los procedimientos y ritmos de una institución universitaria.

\section{La colaboración entre las instituciones universitarias de la Compañía}

La experiencia de la comisión formada en noviembre de 2002 y que ha funcionado a lo largo de un curso es valorada muy positivamente por sus miembros. Ha servido para conocer lo que hacen los distintos centros, establecer colaboraciones en proyectos concretos, intercambiar experiencias y unificar criterios. Fruto de los encuentros que se han celebrado en estos meses es el presente documento.

A partir de dicha experiencia se propone que esta comisión se mantenga, no sólo como un vínculo más que refuerza el proyecto integrado de UNIJES, sino por su capacidad para garantizar unos objetivos deseables para todos los centros, tales como:

1. Formulación de políticas comunes a todos los centros, de acuerdo con la misión propia de estos.

2. Organización de seminarios y encuentros que ayuden a perfilar mejor nuestras actividades de cooperación.

3. Colaboración en programas académicos conjuntos.

4. Búsqueda de una presencia geográfica equilibrada de nuestra cooperación al desarrollo en los distintos continentes.

5. Elaboración de proyectos comunes, que puedan ser realizados como acción conjunta de varios o de todos los centros.

6. Colaboraciones puntuales en proyectos de un centro concreto.

7. Búsqueda de fondos para la financiación de proyectos.

8. Coordinación de actividades de voluntariado de nuestros alumnos en países en desarrollo.

9. Coordinación en las relaciones con otras redes de la Compañía (AUSJAL y otras).

10. Intercambio de información.

Dicha comisión estaría formada por un representante de cada centro (normalmente, el responsable de la cooperación al desarrollo en él). 